\title{
El incesto a la luz de un análisis de género
}

\author{
Carolina Quirós Ferlini \\ Licenciada en Psicología. Psicóloga en el Instituto de Estudios de Género de la Universidad Estatal a Distancia, UNED y \\ psicoterapeuta. Correo electrónico: caroquiros86@gmail.com
}

Recibido: 22 de enero 2013 - Aprobado: 27 de junio 2013

\section{RESUMEN}

La identidad de género, la discriminación, la violencia y la adopción de conductas que se asumen como "naturalmente dadas" y modeladas son factores coadyuvantes en la problemática del incesto. En esta dinámica se identifica la relación existente entre la variable género, las conductas aprendidas de poder y sumisión así como su clara expresión en las manifestaciones sociales de la sexualidad.

Palabras clave: Incesto, análisis, género, violencia.

\section{ABSTRACT}

Gender identity, discrimination, violence and the adoption of behaviors that are assumed "naturally given" and modeled, are contributing factors in the problem of incest. The dynamics of incest identifies the relationship between the variable gender, behaviors learned of power and submission and its clear expression in the social manifestations of sexuality.

Key words: Incest, analysis, gender violence.

\section{Introducción}

Cada sociedad y persona tienen su propia concepción de género. Es así como al nacer, por medio de la observación e imitación de quienes creemos nuestros iguales, adoptamos comportamientos y formas de pensar característicos a nuestra identidad de género, entiéndase esto como la expresión de lo femenino o lo masculino.

La identidad de género, entonces, se convierte en parte de nuestra visión del mundo, historia y tradiciones;

Toda concepción de género se conjuga con las otras visiones que conforman las identidades culturales y las de cada persona, los valores imperantes y las motivaciones para la acción. Así se integran las cosmovisiones de género, sociales y personales. Las ideas, los prejuicios, los valores, las interpretaciones, las normas, los deberes y las prohibiciones sobre la vida de las mujeres y los hombres conforman las cosmovisiones particulares de género, que son siempre etnocentristas (Cazes, 1998: 41).

Es entonces que, lo antes mencionado, se torna de suma importancia a la hora de abordar el tema del incesto, ya que este, por sus características, se esgrime como un delito ligado estrechamente a cuestiones de género que atraviesa a la persona en toda su integridad a nivel físico, social y psicológico y que, en su haber y accionar, 
se encuentra estrechamente ligado a la posición de poder generalmente manifestada por el sexo masculino, el cual por medio de la socialización patriarcal y dentro de un esquema de pensamiento disfuncional se cree con el derecho de poseer tanto el cuerpo como la propiedad física de quienes están bajo su "mando".

Con el objetivo de dar inicio al análisis del delito del incesto bajo una perspectiva de género es preciso realizar un breve repaso por los inicios de la humanidad y el nacimiento del patriarcado.

La primacía masculina no nació junto con la humanidad, sino que surge después de miles de años que la población se congregó, ante todo, alrededor de las madres. Este se distingue por características determinadas por la trasmisión patrilineal del parentesco. Martín-Cano (2000), hace referencia al tema como una estructura social en la cual la autoridad máxima es el padre, siéndolo también el hijo o ascendiente varón mayor de edad y esta se convirtió en una unidad religiosa, legal y económica rígidamente estructurada. El padre de familia contaba con todos los derechos legales, ya que era la única persona reconocida por la ley y quien podía tener todos los bienes familiares.

Pérez (s.f.), contempla el mando varonil como el desencadenante de la violencia de género y lo explica de la siguiente manera:

\section{Cualquier sistema ideológico autoritario $-y$ el sistema patriarcal lo es en grado sumo-necesita transmitir sus postulados de manera incuestionable con el fin de que se puedan sostener como verdades absolutas el conjunto de principios y valores en los que se inspira. En el sistema patriarcal la diferen- cia sexual se presenta como razón suprema, base y fundamento de la discriminación que inspira su ideología. Deja de ser discriminatorio aquello que viene a ser impuesto por fuerza de la naturaleza. Lo biológico queda erigido en destino fatal. Rebatirlo supone un desafio contra natura, algo condenado al fracaso (p.3).}

Es así como, al imponerse el patriarcado y sus postulados, tanto la mujer como los niños, niñas y adolescentes pasaron a ser propiedad del varón.
A nivel nacional, la situación de incesto que viven y han vivido muchos niños, niñas y adolescentes puede ser explicada, sin limitarse a esta única opción, partiendo del desarrollo históricolegal del cuidado de las personas menores de edad. En el ámbito legal, antes de 1979, las personas menores de edad se encontraban bajo un paradigma de situación irregular, el cual ofrecía una visión errónea de los niños; menores antes que personas es decir, eran objetos de cuidado y propiedad de los padres. Por tanto, ellos podían, si así lo creían necesario, educar a sus hijos por medio de la violencia física, entre otros métodos, situación que fue modificada aquel año cuando se reconoció por primera vez a los niños, niñas y adolescentes como personas poseedoras de derechos. En 1983, se creó la Convención Internacional de los Derechos del Niño, reconocida por Costa Rica en 1989 y que da pie a la creación del Código de Niñez y Adolescencia en 1998 (Cartín, comunicación personal, 27 de mayo, 2009).

Hoy, aunque el tema de la protección se encuentra con más presencia en la sociedad, se siguen produciendo denuncias por abuso sexual infantil. La problemática ha incrementado considerablemente a lo largo de los años y las estadísticas así lo confirman. Según Batres (1997), en el Hospital Nacional de Niños (HNN), entre 1992 y 1995 se atendieron 695 niños y niñas víctimas de abuso sexual. De 1990 a 1993, 8,3\% de los niños fueron abusados una sola vez de los cuales $17,2 \%$ eran menores de 3 años; $12,1 \%$ de 3 a 7 años; un $7,7 \%$ de 8 a 12 años; $3,2 \%$ de 13 a 17 años; $20,7 \%$ se negó a hablar acerca de la duración de la situación de abuso sexual y 29,4\% desconocía la duración. Los datos más recientes emitidos por el Patronato Nacional de la Infancia (PANI) exponen que durante el 2011, específicamente el periodo de enero a noviembre, se atendieron 30,640 casos de los cuales 595 refieren a situaciones de abuso sexual intrafamiliar. (Patronato Nacional de la Infancia, 2011, citado por Quirós, 2012)

La permanencia del incesto es facilitado por el silencio, el secreto y la amenaza, lo cual Benavente (2011) apoya y afirma: 
Es difícil la detección del abuso sexual, debido a que no suele dejar marcas fisicas visibles. Debemos observar si presenta cambios bruscos del comportamiento, como la falta de apetito, miedos, insomnio $y$, sobre todo, si presenta conductas sexualizadas, como puede ser la masturbación compulsiva, un lenguaje sexual explícito o juegos sexuales inapropiados para la edad (párr. 1).

Aunque el incesto puede ser abordado desde casi cualquier corriente filosófica y psicológica, el presente artículo se centra en un análisis génerosensitivo por los factores particulares que han influido en la aceptación y perpetuación de la problemática del incesto.

En la actualidad, la violencia de género es un crimen, un delito universal y es penado por la ley, muestra de ello son las diferentes leyes y normativas que a nivel nacional e internacional se han elaborado con el único objetivo de defender los derechos inalienables tanto de las mujeres, los niños, niñas y adolescentes, ejemplo de esto son: la Convención Internacional de los Derechos del Niño, El código de niñez y adolescencia de Costa Rica, mencionados anteriormente, la ley $\mathrm{N}^{\mathrm{o}} 7586$, contra la violencia domestica de Costa Rica, la ley 8590 denominada Ley para el fortalecimiento de la lucha contra la explotación sexual de las personas menores de edad de Costa Rica, el programa ONU mujeres para la Igualdad de Género y el Empoderamiento de la Mujer, entre otros.

La violencia contra las mujeres, las niñas y los niños en el ámbito doméstico está reconocida como un problema mundial significativo. El programa de la Organización de las Naciones Unidas ONU hace referencia a esto y declara:

(...) las desigualdades entre los géneros están muy arraigadas en las sociedades. Las mujeres no tienen acceso a un trabajo decente y se enfrentan a la segregación ocupacional y a las diferencias en los salarios por su sexo. A veces también se les niega el acceso a la educación básica y a los servicios de salud. Las mujeres de todas las regiones del mundo son víctimas de violencia y de discriminación y están mal representadas en los procesos de la toma de decisiones (2011, párr.9).
Actualmente se vive en una sociedad sexista, en donde las conductas violentas contra las mujeres están inscritas en un sistema de relaciones de poder/subordinación entre géneros que abarcan toda la sociedad. Lo que muchos no entienden, en ocasiones se limita únicamente a términos, diferencias mínimas como por ejemplo el sexo y el género; por lo tanto, es necesario dar explicaciones teóricas a términos que pueden ser tan convencionales y naturales en nuestro diario vivir que pocas veces somos capaces de ver sus amplias diferencias.

\section{Desarrollo}

\section{Sexo y Género}

Por sexo se entiende las diferencias entre el macho y la hembra, como una categoría física y biológica, con funciones de reproducción especificas de cada uno. Mientras que género, es una pauta construida social y culturalmente. Según Crooks y Baur (2000), citados por Quirós (2012):

Con sexo aludimos a nuestra feminidad o masculinidad biológica. Hay dos aspectos del sexo biológico: el sexo genético, el cual se determina por nuestros cromosomas sexuales y el sexo anatómico, las diferencias físicas obvias entre hombre y mujeres. El género es un concepto que engloba significados psicosociales especificos agregados a la masculinidad o feminidad biológica. Así, aunque nuestro sexo se vincula a diversos atributos físicos (cromosomas, pene, vulva, etc.), nuestro género se refiere a las características psicológicas y socioculturales asociadas a nuestro sexo; en otras palabras, nuestra feminidad o masculinidad (p.42).

Es a través de estos conceptos que las personas se reconocen cuando se encuentran una frente a otra persona y "hacen suposiciones basadas en la masculinidad o feminidad acerca de cómo es probable que se comporte (Crooks y Baur, 2000; p.43)" (Quirós, 2012).

\section{Identidad y rol de género}

Estos son otros conceptos importantes: 
El primero es "la percepción subjetiva de cada persona de si es hombre o mujer" (Crooks y Baur, 2000, p. 43) y no necesariamente debe coincidir con su sexo biológico. El segundo se refiere a "una serie de actitudes y conductas que en una cultura se consideran normales y apropiadas para los individuos de un determinado sexo" y es lo que se conoce como conducta femenina o masculina (Quirós, 2012: 55).

Entonces, como lo menciona Burin y Meler (1998), citado por Quirós (2012: 56), "la idea general mediante la que se diferencia "sexo" de "género" es que el sexo queda determinado por la diferencia sexual inscrita en el cuerpo, mientras que el género se relaciona con los significados que cada sociedad le atribuye (p.19).”

\section{Femenino y masculino}

Esta construcción de lo que significa ser femenino y masculino, como ya se ha mencionado anteriormente, ha sido hasta ahora mayormente una construcción varonil que ha asignado roles de comportamiento a la mujer. En palabras de Virginia Wolf;

¿Tenéis alguna noción de cuántos libros se escriben al año sobre las mujeres? (...) Me puse a recorrer con desesperación la larga lista de títulos. Hasta los títulos de los libros me hacían reflexionar. Era lógico que la sexualidad y su naturaleza atrajera a médicos y biólogos, pero lo sorprendente y difícil de explicar es que la sexualidad femenina -es decir, las mujeres- también atraen a agradables ensayistas, novelista de pluma ligera, muchachos que han hecho una licencia, hombres que no han hecho ninguna licencia, hombre sin más calificación aparente que la de no ser mujeres. Algunos de los libros eran, superficialmente, frívolos y chistosos; pero, muchos, en cambio, eran serios y proféticos, morales y exhortadores. (...) Mirara uno donde mirara, los hombres pensaban sobres las mujeres y sus pensamientos diferían (Quirós, 2012: 58).

La sensible reflexión de Virginia Wolf nos hace pensar ien qué momento las mujeres realmente tomarán su vida en sus manos y dirán con convicción y coraje: "aquí estoy y no permitiré nunca más que un hombre $u$ otro ser humano sin importar su género alce la voz para hablar por mí"? Mayobre (2002) citado por Quirós (2012) nos permite ver una luz al final del túnel cuándo expresa:

Sin embargo, en los últimos años la tendencia es hacia un cambio en el que las mujeres cobren protagonismo para hablar por sí mismas como sujeto de enunciación, lo cual supone un cambio de paradigma y de lógica, ya que tradicionalmente se considera que el varón es el sujeto dominante de la especie humana, el de la experiencia y que, tiene la capacidad de nombrar el mundo, representar la realidad y de significar a la mujer.

La mujer, en su rol dictado por la sociedad, no debe expresar su sexualidad de ninguna forma, ya que esto no es adecuado para una dama dentro del concepto de feminidad que se maneja, y si lo hace, será vista como de poca valía tal y como lo expresa el refrán popular "la mujer debe ser deseada y no sobrada", o como lo menciona González (2004) citado por Quirós (2012);

con la doble moral sexual existente se destacó como la vivencia de la sexualidad está marcada por una notable diferencia entre los géneros: las prácticas sexuales enaltecen a los varones, pero desprestigian a las mujeres. Existiría, en consecuencia, una desnarcización de la pulsión sexual femenina. Y ello estaría estrechamente relacionado con la tendencia a vivir de modo asociado sexualidad y amor, así como a experimentar con culpabilidad y vergüenza la expresión libre del deseo sexual (p. 61).

Es así como todo lo anterior se engarza en una serie de mandatos, leyes o reglas que los hombres y mujeres deben de cumplir para ser vistos ante la sociedad como personas comprometidas y respetables de su y posición jerárquica en la sociedad y vida privada. Estos comportamientos específicos impuestos a ambos grupos han sido adoctrinados bajo el término: "mandatos del género".

\section{Mandatos del género}

Históricamente, cada país, cultura, y región tiene sus propias leyes de convivencia, comportamiento, posiciones jerárquicas y sexuales. 
En la sociedad costarricense, la forma de ser y de sentirse mujer viene determinada por un estereotipo de 'feminidad' tradicional que, entre otros rasgos que la definen, atribuye una importancia fundamental a valores ligados con lo emocional o relaciones interpersonales; de estas últimas se espera y se fomenta que se lleven a cabo y dentro del seno familiar por lo que es sometida al ámbito del hogar. Otros factores son el afecto, los cuidados y el apego. Sin embargo, no solo se fomenta la creación de estos vínculos, sino igualmente su permanencia; a propósito, Batres (1997) argumenta lo siguiente:

Histórica y culturalmente han sido asignados papeles a los hombres y a las mujeres (...) Además, los roles son los derechos y obligaciones que implican una posición social y genérica; estos informan a las personas acerca de las conductas que se esperan de ellas. Por ejemplo, la posición genérica de madre nos impone determinadas obligaciones y derechos diferenciados a los derechos y obligaciones que tiene la posición genérica del padre (p.4).

De esta manera, lo emocional queda sobredimensionado para las mujeres, al igual que el impacto ante las pérdidas amorosas y la dependencia de los objetos de amor. Es innegable la importancia que adquieren las relaciones humanas, los vínculos de gran intimidad y la permanencia en el tiempo en la organización de la identidad femenina. Por ello, se sienten fracasadas como seres humanos ante las rupturas amorosas o las pérdidas (Dio Bleichmar, 1991, citado por Romero, 2004).

Muchas veces, el discurso familiar y social se encargan de orientar a la mujer para que salga en busca de su propia valoración e identidad fuera de su estirpe. Su propio sostén y el cuidado de los otros pasan a ser su prioridad como objetos que le confieren identificación y valor.

En la amenaza de la pérdida amorosa, las mujeres no solo se sienten responsables de conservar estas relaciones y, por tanto, fracasadas si no lo consiguen, sino que, además, pueden tener depositada en esa meta su valoración integral como personas (Romero, 2004).
Por consiguiente, como lo he mencionado anteriormente, desde el abordaje de la problemática del incesto sin consideraciones de género, se debe tomar en cuenta tanto la dificultad de la socialización femenina como el dominio masculino, desde el cual se generan muchos de estos actos. Es imposible obviar que desde la concepción del patriarcado, las mujeres, los niños, las niñas y los adolescentes son objetos de uso y placer para la figura masculina.

El hecho fundamental en la construcción del patriarcado es el que las mujeres hemos sido, a través de los tiempos, utilizadas como mercancía intercambiable o vendible, así como sus hijas (os). Para lograr la cosificación de las mujeres, se objetiviza y norma su sexualidad y capacidad reproductiva: asi se estableció el poder de los hombres sobre la sexualidad femenina y su función reproductora (Batres, 1997: 8-9).

El reflejo del patriarcado puede observarse en las víctimas y sus madres, pues dentro de toda la situación de revelación del acto de abuso se trata de justificarlo, omitirlo o 'explicarse' a sí mismas que lo sucedido es normal, sobre todo en las víctimas primarias. Millet (1995, citado por Batres, 1997) dice: "La firmeza del patriarcado se asienta también sobre un tipo de violencia de carácter marcadamente sexual, que se materializa plenamente en la violación" (p.9).

Es así como todo lo anteriormente expuesto nos da los elementos necesarios para hilar la problemática del incesto a las diferencias y manifestaciones de género a las que día a día nos vemos expuestos la mayoría del tiempo sin ser conscientes de ello.

\section{Consideraciones de género y el incesto}

Partiendo de una visión génerosensitiva, para el análisis del problema, es importante aclarar que el incesto perpetrado por figuras de autoridad masculinas en contra de niñas está ligado a fuertes raíces de género. La identidad de género, la discriminación, la violencia y la adopción de conductas que se asumen como 'naturalmente dadas' y modeladas son factores coadyuvantes en 
la problemática de la transgresión. Meza y Mata (2003) indican que "En la dinámica de incesto se identifica la relación existente entre la variable género, las conductas aprendidas de poder y sumisión y su clara expresión en la manifestación social de la sexualidad" (párr. 1).

A pesar de que, en su mayoría, los actos incestuosos son realizados a través de manipulaciones, engaños, amenazas y en pocas ocasiones se escucha que en estos haya mediado la fuerza física como una forma de someter a las sobrevivientes, continúan siendo un delito violento que genera grandes consecuencias a quien es presa de este maltrato. Es por eso, que mucho de lo explicado a continuación por Meza y Mata (2003), utiliza el término violencia:

El estudio de la violencia como construcción social supone abordar el concepto de socialización en las dimensiones de poder, sexo y género, y como factores coadyuvantes a la manifestación de la violencia.

El poder se ejerce de forma concreta según circunstancias de tiempo, lugar e ideología y diferenciada por normas y valores establecidas en las relaciones sociales (párr. 5-6).

El género, es la categoría que explica la "dicotomía sexual que es impuesta socialmente por medio de roles y estereotipos que hacen aparecer a los sexos naturalmente desiguales y diametralmente opuestos. Mientras que el sexo, podría decirse que se refiere al orden de lo fisiológico, el género es una construcción social" (Córdoba, 1997: 53-54).

La construcción social de lo femenino y lo masculino además, el papel que tienen mujeres y hombres en la sociedad se aprenden como parte de un proceso que se inicia en la infancia por causa de las relaciones primarias y que, paralelamente, es reforzada en todas las instituciones sociales.

La socialización entendida como un aprendizaje social dinámico, que implica una constante interacción y búsqueda de equilibrio entre las necesidades personales y las demandas del ambiente, se constituye en un proceso de vital importancia para la formación de la identidad de género. Concepto que se materializa como algo "que es aprendido, reforzado y sancionado dentro de la sociedad patriarcal. Lleva el aprendizaje de normas que informan a las personas de lo obligado, lo permitido y lo prohibido" (Navas, 1990: 57).

A través de distintas normas y principios de socialización, las personas aprenden comportamientos masculinos o femeninos que responden a las expectativas sociales e internalizando la justificación de la desigualdad en razón del género.

Razón por la cual a las mujeres se les educa de manera que adopten actitudes de sumisión que se manifiestan por medio de conductas como: dependencia, debilidad, emotividad, poco estímulo para desarrollar su capacidad cognitiva, represión sexual y donde su principal razón de ser se justifica a través de la crianza de los hijos y del cuidado de los otros.

Paralelamente, los estereotipos más difundidos en la construcción genética de lo masculino indican que los hombres representan autoridad, seres libres que gozan de independencia, individualidad, racionalidad, objetividad y sexualmente se les asigna un 'deber ser'.

En la problemática del abuso sexual y, particularmente en las situaciones de incesto, se mencionan una serie de mitos con respecto a la ofendida y el agresor que agudizan la situación de crisis que representa el momento de la revelación. Álvarez (1991) citado por Quirós (2012) expone los siguientes mitos en relación con las víctimas:

1. Tiene fantasías por deseos sexuales reprimidos y miente.

2. Es histérica: le gusta seducir con su manera de vestir, de hablar, por los lugares que visita o las horas de salir, entre otros, por lo que estimulan un comportamiento sexual.

3. Es culpable del abuso porque colabora con el ofensor, aceptando sus insinuaciones.

4. Permiten que las agresiones continúen porque "no dicen nada."

Sobres esta base, es necesario recalcar que primeramente son sujetos de protección en quien confían: su padre o madre; que están bajo la 
"protección" de quien le está haciendo daño; ellos no comprenden de manera absoluta qué es lo que está sucediendo y la mayor parte del tiempo son presas del miedo, ya que poseen una confianza auto impuesta pues en su razonamiento aquel o aquella que tanto les ama no les puede hacer daño; además, generalmente tienen temor de hablar por miedo a las amenazas, coacciones o perder el amor de quien les está incestuando.

En cuanto a los mitos instaurados en la población relacionados con los ofensores, Gutiérrez (1992) acota lo siguiente:

1. Los abusadores son personas sin educación, son adictos, desempleados, inmaduros emocionalmente o perturbados mentales.

2. La mayoría de las agresiones sexuales son cometidas por extraños.

3. Las agresiones sexuales son el producto de urgencias sexuales que no pueden ser controladas por el hombre.

Estas propuestas que tratan de explicar la dinámica del abuso sexual se convierten en justificadores y reforzadores que perpetúan el patrón de comportamiento del ofensor, le da herramientas para 'cubrirse' una vez desenmascarado su actuar e imposibilita a la o él sobreviviente en materia de defensión.

\section{Discusión}

Tal como mencioné anteriormente, es imposible obviar que desde la concepción del patriarcado, las mujeres, los niños, las niñas y los adolescentes son objetos de uso y placer para la figura masculina. Esto se da a raíz de que hombres y mujeres albergan en su socialización estereotipos disimiles y específicos a cada género, lo que desemboca en una serie de demandas y expectativas en relación con la forma en que deberían afrontan las diferentes situaciones o traumas.

Uno de los ejemplos más claros es el incesto, cuando el ofensor es un hombre y el sobreviviente lo es también. Es así que, por ejemplo, algunos de los estereotipos más divulgados en la sociedad son los siguientes:
- "Hombre que incestúa a hombre es homosexual y el hombre que ha sido incestuado lo será también"

- "El hombre que ha sido incestuado por una mujer no puede o debe tener consecuencias"

- "Al cumplir el niño cierta edad, debe ir donde las malas"

No es de extrañar, entonces, que para la población masculina hablar del tema del incesto sea complejo y tabú. Mientras que para las mujeres los estereotipos que les circundan son totalmente diferentes, pero igualmente complicados. Por ejemplo, de las mujeres se espera que sean dependientes, pasivas, temerosas, sumisas, débiles, sentimentales:

He aquí una paradoja: las cualidades que se esperan de las mujeres son, al mismo tiempo, socialmente desvalorizadas (OPS-OMS, 1991). La socialización y el tratamiento desigual favorecen el desarrollo de relaciones de inequidad, dominación, desigualdad y violencia contra las mujeres por parte de los hombres en sociedades patriarcales como la costarricense (Escalante, 2004, párr. 13).

En este caso, lo fundamental es entender que así como para la población masculina una parte importante del foco de atención es la asunción de estereotipos relacionados con su identidad sexual y las consecuencias, especialmente sociales. En cuanto a las mujeres, toma relevancia el control, no solo de sus sentimientos y otros factores, sino la forma en que se ha visto afectada su sexualidad de cara a la sociedad.

En este sentido, la mujer en su rol dictado por la sociedad, no debe expresar su sexualidad de ninguna forma, ya que esto no es adecuado. Aquella que lo haga dentro del concepto de feminidad que se acepta, será vista como una mujer de poca valía lo cual es expresado en el refrán popular "la mujer debe ser deseada y no sobrada" o, como lo menciona González (2004):

(...) las prácticas sexuales enaltecen a los varones, pero desprestigian a las mujeres. Existiría, en consecuencia, una desnarcización de la pulsión sexual femenina. Y ello estaría estrechamente relacionado 
con la tendencia a vivir de modo asociado sexualidad y amor, así como a experimentar con culpabilidad y vergüenza la expresión libre del deseo sexual (p. 2).

Por tal motivo, uno de los sentimientos más difíciles de abordar para la mujer es el haber sentido algún tipo de placer físico con el incesto, lo que no es extraño pues el cuerpo cuenta con infinidad de receptores nerviosos que, aunque se desee, no siempre se pueden 'apagar'.

Finalmente, partiendo de las reflexiones y aportes de profesionales en el tema, queda develado el trasfondo sexo-género-social que se ha consolidado con el paso de los años con el silencio, la perpetuación del incesto y la 'aprobación' social de estereotipos fuertemente arraigados al desarrollo psico-físico-social de todo ser humano.

Dicho en otras palabras, la sociedad a lo largo de los tiempos se ha encargado en asignar a cada persona, hombre o mujer una serie de pautas de comportamiento, formas de pensamiento y maneras de actuar específicas acordes a su sexo y género frente a las diferentes adversidades a las que nos enfrentamos, no es de extrañar entonces que ante problemáticas como la del incesto a través de los años la pauta social haya sido la de callar y aceptar con resignación el maltrato del que se es víctima.

\section{Referencias}

Batres, G. (1997). Del ultraje a la esperanza. Tratamiento de las secuelas del incesto. ( $2^{\mathrm{a}}$. Ed, rev.). Programa Regional de Capacitación contra la Violencia Doméstica. San José: ILANUD.

Burin, M. y Meler, I. (1998). Género y familia: Poder, amor y sexualidad en la construcción de la subjetividad. Buenos Aires: PaidósCrooks, R. y Baur, K. (2000). Nuestra Sexualidad. México: Thomson.

Cazes Menache, Daniel. (1998) La Perspectiva de Género. Guía para Diseñar, Poner en Marcha, dar Seguimiento y Evaluar Proyectos de Investigación y Acciones Públicas y Civiles. Mexico: Conapo-Conam.

Corsi, J. (1989). Violencia Familiar: Una mirada interdisciplinaria sobre un grave problema social. Facultad de Psicología Universidad de Buenos Aires. Buenos Aires.
Escalante, A.C.(2004) Perspectiva social de género. Catedrática Universidad de Costa Rica. Recuperado el 17 de abril del 2012 de: http://www.crid.or.cr/cd/CD_CNE/pdf/spa/ doc1063/doc1063-b.pdf

Gutiérrez, I. (1992). Representaciones Sociales de la Masculinidad. Tesis sometida a la consideración de la Comisión Programa de Estudios de Licenciatura en Psicología. Ciudad Universitaria Rodrigo Facio. San José, Costa Rica.

Martín-Cano, F. (2000) Del matriarcado al patriarcado. Recuperado 21 de mayo de 2012, de: http://www.mujerpalabra.net/pensamiento/franciscamartincanoabreu/ matriarcadoapatriarcado.htm

Mayobre, P. (2002). Repensando la feminidad. Igualmente diferentes. Congreso Nacional de Educación en Igualdades. 153-176. Recuperado el 6 de Septiembre del 2006 de http://webs.uvigo.es/pmayobre/textos/repensando_la_ feminidad_acabado.doc

Meza, L., Mata, L. (2003) Consideraciones sobre la socialización de género y su influencia en la dinámica del abuso sexual. Recuperado 21 de mayo de 2012, de: http://www. ts.ucr.ac.cr/binarios/pela/pl-000115.pdf

Navas, M.C. (1990). Conceptualización de Género. Mujeres Centroamericanas ante la Crisis, la Guerra y el Proceso de Paz. FLACSO-UNICEF.

ONU MUJERES, Entidad de las Naciones Unidas para la Igualdad de Género y el Empoderamiento de las $\mathrm{Mu}$ jeres (2011) Acerca de ONU Mujeres. Recuperado 12 de febrero de 2012, de: http://www.ts.ucr.ac.cr/binarios/ pela/pl-000115.pdf

Pérez, (s.f.) El sistema patriarcal, desencadenante de la violencia de género. Recuperado 21 de mayo de 2012, de: http:// www.projusticia.es/ley $\% 20 \mathrm{de} \% 20$ igualdad/documentos/ la $\% 20$ ignorancia, $\% 20$ esa $\% 20$ atrevida $\% 20$ perez $\% 20$ del $\% 20$ campo $\% 20$ curso $\% 20 \mathrm{a} \% 20$ jueces.pdf

Quirós, C. (2012) El síndrome de Estocolmo como una propuesta teórico-explicativa de la perpetuación del incesto en niñas. San José. Tesis para optar por el grado de Licenciatura en Psicología. Universidad Católica de Costa Rica.

Romero, I. (2004). Develar la violencia: una intervención para la prevención y el cambio. Dirección General de la $\mathrm{Mu}$ jer. Comunidad de Madrid. Papeles del Psicólogo, 88. Recuperado el 7 de noviembre del año 2010 de: http:// www.papelesdelpsicologo.es/vernumero.asp?id=1156

Tamés, M. (1999). La mujer y la crisis de la familia. San José, Costa Rica: Ediciones Promesa. 